\title{
Concomitant diagnosis of asthma and allergic bronchopulmonary aspergillosis in a previously healthy 26-year old Afghani male
}

Fotis Sampsonas ${ }^{1}$, Matthaios Katsaras ${ }^{1}$, Ourania Papaioannou ${ }^{1}$, Theodoros Karampitsakos ${ }^{1}$, Loukas Kakoullis ${ }^{2}$, Argyrios Tzouvelekis $^{1}$

\section{INTRODUCTION}

Allergic bronchopulmonary aspergillosis (ABPA), caused by A. fumigatus, occurs in patients with a history of asthma or cystic fibrosis. The diagnosis of asthma usually precedes ABPA for over 10 years ${ }^{1,2}$. We present a case of concomitant diagnosis of ABPA and asthma in a 26-year-old immigrant from Afghanistan.

\section{METHODS}

A 26-year-old male presented with a 3-week history of dyspnea on exertion, chest tightness and productive cough with excretion of copious purulent secretions. The patient did not report fever, weight loss or haemoptysis. He had no history of asthma or atopy. Physical examination revealed a respiratory rate of 19/min and SpO2 of 95\% on room air, polyphonic wheezing, crackles in middle lung zones and significant digital clubbing.

\section{RESULTS}

Laboratory results were significant for an eosinophil count of 2350 cells/ $\mu \mathrm{L}$ and IgE levels were above the measurable amount of $10.000 \mathrm{IU} / \mathrm{mL}$. Sputum Gram and Ziehl-Neelsen stains were negative. PPD reaction was 16 $\mathrm{mm}$. High resolution CT of the chest revealed bilateral cylindrical and cystic bronchiectasis with finger-in-glove sign, mucous plugs, and a tree-in-bud pattern (Figure 1). Spirometry revealed an obstructive profile with a postbronchodilation FEV1 of 55\%, FEV1/FVC of $67 \%$ and reversibility above $12 \%$ and $200 \mathrm{~mL}$. BAL Ziehl-Neelsen stain and PCR for tuberculosis were negative. Direct microscopy revealed the presence of hyphae, confirmed to be $A$. fumigatus (Figure 2). Specific Aspergillus IgE and IgG titers were increased. The patient received oral corticosteroids and itraconazole; his symptoms improved, and he was discharged.

\section{CONGLUSIONS}

Although a diagnosis of asthma usually precedes ABPA for more than a decade ${ }^{1}$, this case demonstrates that ABPA and asthma can present concomitantly in patients with unremarkable past medical history.

Figure 1. High resolution CT of the chest

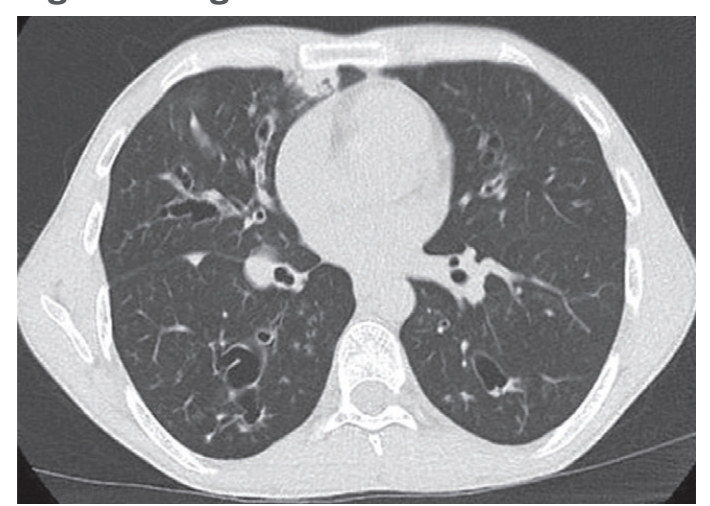

\section{AFFILIATION}

1 Department of Respiratory Medicine, University Hospital of Patras, Patras, Greece 2 Department of Internal Medicine, Nicosia General Hospital, University of Cyprus Medical School, Nicosia, Cyprus

\section{CORRESPONDENCE TO}

Fotios Sampsonas. Department of Respiratory Medicine, University Hospital of Patras, 26504, Rion, Patras, Greece.

E-mail: fsampsonas@gmail.com

\section{KEYWORDS}

asthma, allergic bronchopulmonary aspergillosis

Received: 28 September 2020

Revised: 30 January 2021

Accepted: 14 March 2021

Pneumon 2021;34(1):2

https://doi.org/10.18332/pne/135957 
Figure 2. Direct microscopy revealed the presence of hyphae, confirmed to be A. fumigatus

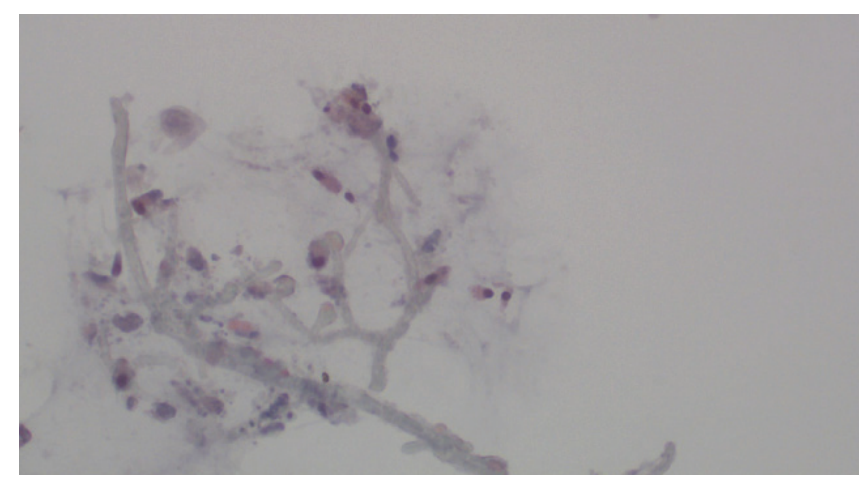

\section{CONFLICTS OF INTEREST}

The authors have completed and submitted the ICMJE Form for Disclosure of Potential Conflicts of Interest and none was reported.

\section{FUNDING}

There was no source of funding for this research.

\section{DATA AVAILABILITY}

The data supporting this research cannot be made available for privacy reasons.

\section{PROVENANGE AND PEER REVIEW}

Not commissioned; internally peer reviewed.

\section{REFERENGES}

1. Shah A. Allergic bronchopulmonary aspergillosis: an Indian perspective. Curr Opin Pulm Med. 2007;13(1):72-80. doi:10.1097/MCP.0b013e328011a284

2. Agarwal R, Chakrabarti A, Shah A, et al. Allergic bronchopulmonary aspergillosis: review of literature and proposal of new diagnostic and classification criteria. Clin Exp Allergy. 2013;43(8):850-873. doi:10.1111/cea.12141 